\title{
Que(e)re Gegenübertragung? Zur (Ir-)Relevanz sexueller Differenzen in der klinischen Praxis
}

\author{
Nicole Burgermeister und David García Núñez (Zürich)
}

\begin{abstract}
Zusammenfassung: Anhand eines Beispiels aus einer Gruppentherapiesitzung beleuchten die Autor_innen die klinische Praxis als Ort, an dem heteronormative Dynamiken reinszeniert wie zugleich stets auch polymorph-pervers durchque(e)rt werden. Unter Einbezug einer queeren Perspektive werden die von der Psychoanalyse reproduzierten gesellschaftlichen Normalitätsfolien und (homophoben und cisnormativen ${ }^{1}$ ) Abwehrmechanismen kritisch beleuchtet. Dabei wird der Schwerpunkt auf die Auseinandersetzung mit der Gegenübertragung gelegt.
\end{abstract}

Schlüsselwörter: Heteronormativität, Queer Studies, Gegenübertragung, Gruppenpsychotherapie, homophobe und cisnormative Abwehr

«Was soll dieses Geplauder bringen?» Herr S., ein kräftiger, 55-jähriger Mann mit breitem sorgfältig gepflegten Schnauz, der vom Aussehen her eher in einen Mafiafilm als in die Psychotherapeutische Tagesklinik zu passen scheint, sitzt, wie meist in der Gruppenpsychotherapie, die ich (N.B) zweimal pro Woche in der Tagesklinik leite und an der zu diesem Zeitpunkt 6 Frauen und 5 Männer teilnehmen, breitbeinig und mit herausforderndem Blick mir direkt gegenüber. Eingetreten mit der Diagnose einer Erschöpfungsdepression nach einem Konflikt mit seinem Vorgesetzten, hat Herr S. - verheiratet und Vater von zwei Kindern bereits nach kurzer Zeit die Rolle des - wie er sich selber nennt - «Gruppenpapas» eingenommen. Zu dem Zeitpunkt ist die Gruppe sehr fokussiert auf die psychosoziale Not mehrerer weiblicher Gruppenmitglieder, die das Gefühl haben, «sich im Kreis zu drehen». Die Männer, allen voran Herr S., sind damit beschäftigt, den Mitpatient_innen² Ratschläge zu geben, während das Behandlungsteam aus seiner Sicht «die Leute mit ihren Problemen allein lässt» und, wie er mir einmal direkt vorwarf, «nicht zupackt». Auf die Versuche des Teams, sich gemeinsam mit ihm mit seinen Schwierigkeiten auseinanderzusetzen, reagiert Herr S. gekränkt und insbesondere den weiblichen Teammitgliedern gegenüber häufig entwertend. Als Gruppentherapeutin finde ich mich in der unangenehmen Position der von 
der Gruppe als kalt, enttäuschend und gleichgültig erlebten Mutter wieder, herausgefordert durch die konkurrierende bis entwertende Haltung, die Herr S. mir gegenüber einnimmt. Es ist in einer von mir als besonders anstrengend erlebten Therapiesitzung, in der ich angesichts der anhaltenden Unzufriedenheit der Gruppe und der wiederholten «Attacken» von Herrn S. zunehmend mit eigenen Insuffizienzgefühlen hadere, als ich mich plötzlich mit einer in meinem Erleben lustvollen Fantasie konfrontiert sehe: Herr S. und ich als zwei miteinander ringende, ineinander vergrabene und sich im Sand wälzende Männer, in ebenso aggressiver wie erotischer Umarmung, es sozusagen «heftig miteinander treibend». Ich verspüre Lust, mich wie Herr S. breitbeinig auf den Stuhl zu setzen. Mehr noch: Ich nehme einen Moment lang eine starke sexuelle Erregung in mir wahr, sowie den Impuls, "es ihm schon zu zeigen» und mich mit ihm, auf ihn, auf den Boden zu werfen. Zunächst bin ich etwas irritiert über diese so unvermittelt in meiner Gegenübertragung auftauchende «schwule» Fantasie. Weder bin ich ein Mann, noch ist Herr S. homosexuell, noch habe ich selber ihn bisher als erotisch anziehend erlebt. Und besonders «phallisch» fühle ich mich in der beschriebenen Gruppensituation auch nicht gerade. Ich stelle mir vor, wie irritiert viele meiner Teamkolleg_innen wären, wenn ich ihnen in der Nachbesprechung von meiner Fantasie erzählen würde. Etwas beschämt bin ich geneigt, dieses innere Bild sogleich wegzuschieben.

\section{Zur Debatte um die Gegenübertragung}

Zur Übertragung, von Laplanche und Pontalis (1967) im «Vokabular der Psychoanalyse» definiert als der «Vorgang, wodurch die unbestimmten Wünsche an bestimmten Objekten im Rahmen eines bestimmten Beziehungstypus, der sich mit diesen Objekten ergeben hat, aktualisiert werden», existiert in der Psychoanalyse eine breite Auseinandersetzung (vgl. u. a. Körner, 1990; Mertens, 1991; Gysling, 1995; Thomä, 1999; Bettighofer, 2000). Diese wird von einer nicht minder ausführlichen Debatte um den Begriff der Gegenübertragung, nach Laplanche und Pontalis verstanden als «Gesamtheit der unbewussten Reaktionen des Analytikers auf die Person des Analysanden und ganz besonders auf dessen Übertragungen», begleitet (vgl. u. a. Thomä, 1999; Zeul, 1999; Hartmann \& Milch, 2000; Kläui, 2008). Beiden Begriffen gemeinsam ist ihre definitorische Elastizität, welche es im Verlauf der Zeit ermöglicht hat, dass sie von den unterschiedlichsten psychoanalytischen Schulen und anderweitigen psychotherapeutischen Richtungen prominent thematisiert und problematisiert, immer wieder re-definiert, aber auch produktiv verwendet wurden. Vom heutigen Standpunkt aus wesentlich ist unseres Erachtens die Betonung 
des interaktionellen Charakters der Übertragungs-Gegenübertragungsdynamik, insofern als sie sowohl von den - trotz stattgefundener Lehranalyse stets vorhandenen - (rest-)neurotischen Konflikten und Übertragungsneigungen der Analytiker_innen als auch von deren emotionalen Antworten auf die spezifischen Interaktionsdynamiken mit den jeweiligen Patient_innen bestimmt ist. Insofern gehen wir daher davon aus, dass «Übertragung und Gegenübertragung gemeinsam ein intersubjektives System reziproker, gemeinsamer Einflussnahme» (Stolorow et al.,1996, S. 65) bzw. ein «intersubjektives Erfahrungsfeld, in dem sich Übertragung und Gegenübertragung wechselweise verschränken» (Mertens, 1991, S. 49), bilden. Hinsichtlich der Funktion der Gegenübertragung bewegt sich die Diskussion im Spannungsfeld zwischen einer Sichtweise, welche mit Bezugnahme auf Freud die Gegenübertragung primär als einen auf die Neurose der Analytikerin zurückzuführenden und entsprechend zu überwindenden Störfaktor versteht, und einer Perspektive, welche in der Gegenübertragung die direkte (produktive) emotionale Resonanz des Unbewussten der Patientin in der Analytikerin sieht. Letztere Position knüpft an die von Heimann vertretene These von der Gegenübertragung als "the patient's creation" an, mit der sie die Gegenübertragung als "emotional response" des Analytikers auf seinen Patienten und als "one of the most important tools" seiner Arbeit verstand (Heimann, 1950). Während dieser "emotional response" bis in die 1950er Jahre wenig therapeutischer Nutzen beigemessen wurde, wird heute von manchen Autor_innen (vgl. z. B. Kläui, 2008) eine gegenläufige Entwicklung kritisiert, nämlich ein unreflektiertes kausallogisches Kurzschliessen von Gefühlsreaktionen des Analytikers auf unbewusste Zustände der Patientin.

Als affektives Amalgam entzieht sich die Gegenübertragung letztlich einer eindeutigen Verortung ihrer eigenen Herkunft. ${ }^{3}$ Die Gefühle des Analytikers sind Christian Kläui zufolge «bestenfalls Indikatoren», die «anzeigen, dass etwas Merkwürdiges, Befremdendes im Gang ist» (2000, S. 199), welches der Analytiker «gerade nicht verstehen kann und dessen Nicht-Verstehen» er auszuhalten hat.

\section{«Schwule Gegenübertragung» in der Gruppentherapie}

Merkwürdig erschien mir (N.B) im eingangs geschilderten Beispiel meine lustvolle Fantasie in der Tat: War es nicht etwas unpassend, mitten in der Gruppentherapie an Sex zu denken? Und war es nicht besonders absurd, sich die erotische Fantasie zwischen einer Therapeutin und einem heterosexuellen Mann als schwul vorzustellen? Zumal zu dem Zeitpunkt der Fokus in der Gruppe stark auf den Frauen lag, die entweder als «arm und bemitleidenswert» (die Patient_innen) oder als «ungenügend und unfähig» (die weiblichen Teammitglieder) erschienen. 
In den folgenden Therapiesitzungen bin ich als Gruppenleiterin innerlich primär mit dieser - von mir zu diesem Zeitpunkt so interpretierten - «Mutterübertragung» beschäftigt.Zunehmend kommen denn auch seitens derTeilnehmer_innen Gefühle derWut und Enttäuschung über die als unzureichend erlebten eigenen Mütter zur Sprache. Herr S. beteiligt sich weiterhin in erster Linie mit Ratschlägen sowie Kritik am Behandlungsteam. Darüber hinaus bringt er sich nicht mit Eigenem ein. In einer dieser Situationen, als er mürrisch wirkend in der Gruppe sitzt, kommt mir die eingangs geschilderte Fantasie von uns beiden als ringenden Männern wieder in den Sinn. «Wir sind sehr mit den Müttern beschäftigt», stelle ich fest. «Was ist mit denVätern?» Herr S. zuckt zusammen, schweigt erst. Dann meint er: «Der war wenig da.» Die Gruppe hört berührt zu, als der sonst so forsch auftretende Patient mit Tränen in den Augen zu erzählen beginnt, wie sehr er sich manchmal gewünscht hätte, von seinem Vater «geholt und in den Arm genommen» zu werden. Seine vor einigen Sitzungen an mich gerichtete Kritik, dass ich zu wenig «zupacke», fällt mir wieder ein und ich frage mich, ob Herr S. mich möglicherweise ähnlich erlebt wie seinen Vater, der die sich auch körperlich manifestierenden Liebeswünsche seines Sohnes nicht erfüllte, die Familie im Stich liess, während er als Sohn die Rolle desjenigen übernahm, der sich um die anderen kümmern musste.

Ab diesem Zeitpunkt entspannt sich die Dynamik in der Gruppe. Der Fokus liegt nicht mehr so stark auf den Ansprüchen an das Behandlungsteam und den damit einhergehenden Frustrationen. Vielmehr beginnen die Teilnehmer_innen, sich vermehrt jenseits der bis dahin festgefahrenen Rollen füreinander zu interessieren und konfrontieren sich gegenseitig mit Fragen und Feedback zu ihnen selber und ihren Schwierigkeiten im Leben. Dabei fällt auf, dass auch auf der gleichgeschlechtlichen Ebene, zwischen den Frauen ebenso wie zwischen den Männern, eine neue Dynamik entsteht, die mir wie ein lustvoll-herausforderndes «miteinander Raufen» vorkommt. Bei einigen Teilnehmer_innen, u. a. Herrn S., reduziert sich zeitgleich die depressive Symptomatik deutlich.

Wie kann nun diese Veränderung verstanden werden? Und inwiefern spielte die «schwule» Gegenübertragung der Gruppentherapeutin dabei eine Rolle? Im Rahmen unseres gemeinsamen intervisorischen Nachdenkens interpretierten wir die Situation wie folgt: Indem die Therapeutin, was zunächst naheliegend schien, sich auf die Bearbeitung der Mutterübertragung konzentrierte, übersah sie, dass sie für Herrn S. auch - oder vielmehr - eine Vaterfigur repräsentierte. Sie erscheint in der Übertragung des Patienten wie der unnahbare Vater, der die Familie - die Gruppe - im Stich lässt, nicht lustvoll mit ihm kämpft, ihn nicht in den Arm nimmt, seine Wünsche danach, «gepackt zu werden», zurückweist. Erst die Wahrnehmung ihrer 
Gegenübertragungsfantasie ermöglichte es der Gruppenleiterin, den Blick auch auf das zu lenken, was zunächst weniger offensichtlich war: Die Vaterübertragung von Herrn S. auf sie, die Enttäuschung über die unerfüllt gebliebenen Liebeswünsche sowie den lustvollen Anteil an der sowohl bei ihm wie auch insgesamt sich in der Gruppe zeigenden aktiven wie passiven Aggression, die durchaus auch eine homoerotische Komponente beinhaltet. Indem die Therapeutin sich in ihrer Fantasie ebenso mit dieser männlichen Position wie mit dem gleichgeschlechtlich männlichen Begehren identifizieren konnte, entstand ein Raum, in dem die unbewussten Wünsche des Patienten zur Sprache kommen konnten. Zugleich - so unsere These - konnte auf der Gruppenebene die Fokussierung der weiblichen Insuffizienz und die Abwehr auch gleichgeschlechtlich orientierter Zuneigung aufgegeben werden zugunsten einer offenen Haltung gegenseitigen Interesses an- und aufeinander.

Einwenden liesse sich nun, dass die Fantasie, in der die Therapeutin mit ihrem Patienten in einen erotisch-aggressiven Dominanzkampf verwickelt ist, in erster Linie eine (rest-)neurotische Reaktion ihrerseits auf die anspruchsvolle Gruppensituation darstelle, eine Abwehr ihrer eigenen Insuffizienz- und Ohnmachtsgefühle durch die körperliche Vereinigung mit einer potenten Vaterfigur und die Verleugnung der Kastration. Selbst dann würde sich die Frage stellen, warum diese Fantasie gerade in dieser spezifischen Situation mit genau jenem Patienten aufgetreten ist. Denn dass Patient_innen dem Behandlungsteam und dessen therapeutischen Angeboten mit Skepsis bis hin zu Entwertung begegnen, gehört zum teilstationären Alltag. Dass in der Gegenübertragung der Gruppentherapeut_innen dabei sexuelle Fantasien auftauchen, kommt hingegen doch eher selten vor. Eva Poluda-Korte hat in einem Aufsatz, in dem sie die geringe Beachtung, bzw. Tabuisierung sexueller Gegenübertragung in der psychoanalytischen Literatur problematisiert, auf den ihrer Erfahrung nach «hochspezifischen Erkenntniswert» hingewiesen, den die Auseinandersetzung mit solchen zwar selten, jedoch oft plötzlich auftretenden Gegenübertragungsphänomenen habe. Ihr zufolge handelt es sich um einen «Indikator für eine starke Abwehr, die von aufgestauten Triebkräften aktuell durchbrochen wird» (1993a, S. 192) und sie ist überzeugt, dass sie oft «zum Kern der behandelten Störung» (ebd., S. 197) führt. So kann es sein, dass Wünsche, die von einem Patienten nicht toleriert werden können, stattdessen in der Analytikerin untergebracht und so stellvertretend von dieser erlebt werden (ebd., 192f.). Wesentlich ist dabei, ob die Analytikerin in der Lage ist, diese wahrzunehmen und zuzulassen, oder ob sie selber zur Abwehr beiträgt. Hätte sie das sexuelle Moment der negativ ödipalen Übertragung (wie dies in heteronormativ geprägten Vater-Sohn-Beziehungen ebenso wie in der psychoana- 
lytischen Perspektivierung dieser Beziehung häufig geschieht) abwehren müssen, statt es lustvoll zu erkunden, wäre es womöglich zurWiederholung einer Dynamik gekommen, die der Patient bereits kannte: Er wäre mit seinen Liebeswünschen in die heteronormativen Schranken verwiesen worden («Männer lieben keine Männer»), die Therapeutin hätte ihm gezeigt, dass «ihr Phallus» der grössere ist und die gleichgeschlechtlichen Beziehungen wären weiterhin von narzisstisch anmutenden Konkurrenzkämpfen dominiert gewesen.

\section{Bisexuell oder queer?}

Gemäss Almuth Sellschopp (1999, S. 1046) ist die Voraussetzung für erfolgreiches analytisches Arbeiten, «dass eine rigide Etablierung der Geschlechtsidentität im Analytiker soweit wie möglich rückgängig gemacht wird zugunsten der Fähigkeit fluktuierender bisexueller Identifizierungen und ihrer phantasievollen spielerischen Übergänge». Die «psychische» bzw. «basale Bisexualität» (Becker, 2005, S.65) wurde zwar bereits von Freud (1905) beschrieben, das Potenzial seines Konzeptes wurde von ihm allerdings erst teilweise erkannt. Vor dem Hintergrund neuerer Entwicklungen in den Gender und Queer Studies wurde es von verschiedenen Autor_innen neu aufgegriffen (vgl. Quindeau, 2008; Gsell \& Zürcher, 2011; Dannecker, 2005). Die seit den 1990er Jahren im wissenschaftlich-politischen Kontext zunehmend bedeutsameren interdisziplinär ausgerichteten Queer Studies bauen auf den Errungenschaften sowohl der feministischen wie auch der LGBT(Lesbian, Gay, Bisexual and Transgender)-Bewegung auf, problematisieren aber gleichzeitig eine auch dort oft stattfindende Essenzialisierung sozialer Kategorien wie «Mann»/»Frau» oder «hetero-/homo-/bisexuell». Zu den zentralen Anliegen der Queer Studies gehört die Dekonstruktion normalisierender Praktiken um Geschlecht, Sexualität und weiterer «Normalitäten» (wie z. B. «weisse» Hautfarbe oder Nicht-Behinderung). Insbesondere fragen sie auch danach, was mit jenen Menschen geschieht, die in diesen gesellschaftlichen Normalitätsvorstellungen nicht oder bestenfalls als Abweichung repräsentiert sind, pathologisiert, ausgeschlossen oder - auch idealisierend - exotisiert werden, die also que(e)r zu diesen stehen.

Gerade die therapeutische Praxis zeigt, wie wirkmächtig einerseits gesellschaftliche Kategorisierungen für das Leben von Individuen sind, wie beschränkt sich damit aber anderseits die Lebensrealitäten von Menschen erfassen und verstehen lassen und wie Patient_innen - ebenso wie, z. B. im eingangs beschriebenen Beispiel, Therapeut_innen - Normalitätsvorstellungen in Fantasie und Realität immer wieder auch durchkreuzen. Freuds Sichtweise wirkt dabei in vie- 
ler Hinsicht ziemlich «queer», wenn er beispielsweise beschreibt, dass «weder im psychologischen noch im biologischen Sinne eine reine Männlichkeit oder Weiblichkeit» gefunden werden könne, vielmehr jede Person «eine Vermengung ihres biologischen Geschlechtscharakters mit biologischen Zügen des anderen Geschlechts und eine Vereinigung von Aktivität und Passivität» aufweise (2000 [1905], S. 123). Psychoanalytiker_innen hätten es daher mit einer ursprünglichen «Unabhängigkeit der Objektwahl vom Geschlecht des Objekts» (ebd., S. 56) seitens der Analysand_innen zu tun. Umgekehrt könnten aufgrund der ursprünglichen, ausschliesslichen Fixierung der Menschen auf Sexualobjekte die «geschätzten oder verehrten Personen» - sprich: auch Psychotherapeut_innen und Patient_innen «für das Unbewusste in uns immer noch Sexualobjekte» sein (2000 [1912], S. 165). Männlich und weiblich - wie ebenso folgerichtig hetero- und homosexuell - sind aus triebtheoretischer Perspektive so eher als «Positionen» (vgl. Quindeau, 2008; Gsell \& Zürcher, 2011), denn als «natürliche» Entitäten zu verstehen.

Ilka Quindeau weist den Kategorien Homo- und Heterosexualität ebenso wie denjenigen der «Männlichkeit» und «Weiblichkeit» primär eine «gesellschaftliche Ordnungsfunktion» zu: «Für das psychoanalytische Denken ist eine solche Kategorisierung allerdings wenig hilfreich» (2008, S. 265). Aus der Perspektive einer psychoanalytischen Sexualtheorie, die unbewussten Vorgängen das entscheidende Gewicht einräume,

erscheinen die Eckpfeiler unseres Alltagsverständnisses von Sexualität - die Geschlechterdichotomie und der Primat der Heterosexualität - keineswegs selbstverständlich und stehen einem psychoanalytischen Verständnis eher im Wege, wenngleich sie auch in manch eine Theorie der Psychoanalyse mit eingehen. (Quindeau, 2008, S. 288)

Schliesslich macht Martin Dannecker darauf aufmerksam, dass «jede interessante Theorie über Sexualität immer auch eine Verteidigung der Sexualität und der um sie kreisenden Wünsche und Abneigungen ihres Autors bzw. ihrer Autorin» enthält (Dannecker, 2005, S. 81). Vor dem Hintergrund einer queeren Auseinandersetzung mit Sexualität und Geschlecht erscheint uns als Praktiker_ innen deshalb ein Zugang wichtig, der diese Kategorien sowohl auf Patient_innenwie auch Therapeut_innenseite stets einerseits als historisch-gesellschaftlich konstruiert, wie andererseits auch als gesellschaftliche, psychische und körperliche Realität(en) perspektiviert (vgl. Burgermeister \& Garcia, 2015). 


\section{Homophobe und cisnormative Abwehr}

Heinrich Racker hat in seinen Schriften zur Gegenübertragung wiederholt auf die Bedeutung des positiven wie negativen Ödipuskomplexes in der Gegenübertragung des Analytikers hingewiesen. Zumindest theoretisch würden die meisten Analytiker_innen ihm wohl zustimmen, wenn er - in seinem Beispiel bezogen auf den männlichen Analytiker - sagt:

Dem männlichen Patienten gegenüber besteht daher der schlummernde oder wirkende Wunsch, von ihm geliebt zu werden, der Wunsch, dass er sich unterwerfen möge, und noch tiefer finden sich alle passiven und aktiven homosexuellen Regungen. (Racker, 2002 [1948], S. 130)

Dass homoerotische Fantasien, Impulse und Wünsche auf Seiten der Analytiker_in zur Sprache gebracht werden, kommt dennoch - so weit wir das überblicken können - sowohl in Super- und Intervisionen, Fallbesprechungen wie auch der Literatur eher selten vor (Ausnahmen sind z. B. Zeul, 1993; Poluda-Korte, 1993b, 2000; Koellreuter, 2000; Balmer, 2001).Verschiedene Autor_innen verweisen auf die trotz einer vordergründigen Öffnung nach wie vor manifest oder latent wirkmächtige Pathologisierung und Diskriminierung von homosexuellen Analytiker_innen und Patient_innen in der Psychoanalyse, ihren Institutionen und Publikationen (vgl. Rauchfleisch et al., 2002; Langiardi \& Capozzi, 2004; Dannecker, 2007; Quindeau, 2015; Heenen-Wolff, 2015; Gschwind, 2015; Böllinger, 2015). Auf die konkrete Arbeit mit Patient_innen und die Übertragungs-Gegenübertragungsdynamik wirkt sich dies insofern aus, als dass von "long-standing and now well-known heterosexist, heterophilic and/or homophobic countertransference biases in clinical work with and psychoanalytic writing about gay and lesbian individuals" ausgegangen werden muss (vgl. Phillips, 2003, S. 1446).

Die Wirkmächtigkeit der heteronormativen Ordnung und der damit einhergehenden Homophobie hat Auswirkungen nicht nur auf die Arbeit mit bi- oder homosexuellen Patient_innen, sondern auf grundsätzlich jede Beziehung zwischen allen Geschlechtern, ob hetero-, bi-, homo- oder wie auch immer sexuell. Anna Koellreuter etwa hat gezeigt, wie sich homosexuelle Übertragungen und Gegenübertragungen nicht nur im Analyseprozess mit lesbischen Frauen zeigen, sondern grundsätzlich immer in Frau-Frau-Analysen. Die Tendenz besteht, dass Analytiker_innen ihren Analysand_innen aber oft vorwiegend aus einer entsexualisierten mütterlichen Position heraus begegnen und diese Begehrensdimension 
verloren geht, was zu Stagnationen im Analyseprozess führen könne. Sie interpretiert diese als Folge einer «gemeinsamen Angstabwehr von Analytikerin und Analysandin» (2000, S. 13) in der Konfrontation mit dem Sexuellen. Dabei betont Koellreuter, dass homophobe Gegenübertragungsreaktionen auch in gegengeschlechtlichen Analysen auftreten und den analytischen Prozess behindern können:

Homophobie beinhaltet immer auch die Angst vor der eigenen Körperlichkeit. Oder die Angst vor dem eigenen unbewussten Sexuellen. Und dies betrifft beide Geschlechter, ob homo-, hetero- oder bisexuell, ob Analytikerin oder Analysandin. Denn die Garantie einer gesicherten Geschlechtsidentität ist nirgends gegeben. (Koellreuter, 2000, S. 139)

Deutlich wird hier die-auch für die psychoanalytische Theorie-sehr wesentliche enge Verknüpfung von sexuellem Begehren und Geschlechtsidentität, ein Phänomen, das Judith Butler (1990) als «heterosexuelle Matrix» beschreibt. Dabei handelt es sich um ein kulturelles Ordnungsraster, das einen scheinbar natürlichen Zusammenhang von anatomischem Geschlecht (sex), Geschlechtsidentität (gender) und Sexualität nahelegt sowie ein Bild davon zeichnet, was «ein richtiger Mann» bzw. «eine richtige Frau» sei. In meiner sexualtherapeutischen Arbeit mit Männern mit erektiler Dysfunktion etwa bin ich (D. G.) oft mit deren Angst konfrontiert, aufgrund dieser Symptomatik «kein echter Mann» zu sein. Bezeichnenderweise zeigen sich die analogen Ängste bei Patientinnen eher im reproduktions-sexuellen Kontext («Was bin ich für eine Frau, wenn ich keine Kinder bekomme?»). Die heterosexuelle Matrix stiftet Sinn und Ordnung. Psychoanalytisch betrachtet lässt sich jedoch das, was Butler treffend die «Gespenster der Diskontinuität und Inkohärenz» nennt (1990, S. 39), letztlich nie vollständig bannen, es löst Verunsicherung aus, auch bei Behandelnden. Besonders pointiert zeigt sich dies, wenn sich Transpersonen in der psychoanalytischen Praxis vorstellen. Volkmar Sigusch schildert, wie auch erfahrene psychoanalytische Therapeut_innen, mit den Transitionswünschen ihrer Klient_innen konfrontiert, ein vordergründig irrationales Sicherheitsbedürfnis entwickeln, welches dem Wunsch entstammt, der von den Klient_innen erlebten und berichteten Verflüssigung der - zuvor als unhinterfragbar verstandenen - Sexualität und Geschlechtlichkeit mittels Sicherheits- und Ordnungsmechanismen begegnen zu können (Sigusch, 1995). Er führt diese Dynamik auf die sog. "zissexuelle Abwehr» ${ }^{4}$ zurück: «Der transsexuelle Wunsch verwirrt [die Untersuchenden] so 
basal, dass sich eine totalisierende Abwehr, die Grauen und Abweichung bannen will, beinahe reflektorisch einstellt, in psychischer wie in epistemischer wie in diskursiver Hinsicht» (Sigusch, 2011, S. 126). Entspricht die entsprechende Person noch zusätzlich nicht der erwarteten (hetero-)sexuellen Logik, verstärkt sich die entstehende Irritation. Die Verweigerung mancher Klient_innen, parallel zur Transition die Geschlechtlichkeit des Sexualobjektes zu «wechseln», führte bis vor nicht allzu langer Zeit dazu, dass die «Echtheit der Transsexualitätsdiagnose» in Zweifel gezogen wurde. Liebte beispielsweise eine Transfrau - auch nach geschlechtsangleichenden Schritten - nach wie vor Frauen, so durfte sie mit dem Misstrauen des medizinisch-psychiatrisch-psychoanalytischen Systems und mit der Verweigerung von geschlechtsangleichenden Massnahmen rechnen. Angesichts ihres Transitionswunsches und ihrer Abhängigkeit von medizinischpsychiatrisch-psychoanalytischen «Expert_innen» lernten schwule Transmänner und lesbische Transfrauen, unter diesen Umständen schnell über ihre Sexualität zu schweigen (oder zu lügen).

\section{Der Therapieraum als signifikanter Ort}

Aus klinischer Perspektive ist gemäss u. E. die Auseinandersetzung mit den beschriebenen Dynamiken deshalb so wesentlich, weil der therapeutischen Beziehung als einem Raum, in dem hetero- und cisnormative Ordnungen ebenso reinszeniert wie durchkreuzt werden, hochsignifikante Bedeutung zukommt. Jean Laplanche hat die psychoanalytische Situation als «Neuetablierung eines Ortes der Urverführung» (2011, S. 190) konzeptualisiert. In seiner «Allgemeinen Verführungstheorie» beschreibt er Sexualität aus einer - nicht biologistischen triebtheoretischen Perspektive als etwas, was - wie Pierre Passett treffend beschreibt - «genau genommen nicht eine Entwicklung, sondern eine Geschichte ist» (2014, S. 145), entstanden im Rahmen einer sozialen Interaktion, nämlich der «Urverführung» als jener «Grundsituation, in der der Erwachsene - der ursprüngliche Andere - dem Kind sowohl nicht-verbale, verbale als auch verhaltensvermittelte Signifikanten unterbreitet, die von unbewussten sexuellen Bedeutungen durchdrungen sind», den sogenannten «rätselhaften Signifikanten» (Laplanche, 2011, S. 158f.). Quindeau weist darauf hin, dass die hetero- - und wir würden hinzufügen cis- - normative Ordnung in diesem Prozess insofern konstitutiv ist, als das Begehren der elterlichen Bezugspersonen - egal welchen Geschlechts oder sexueller Orientierung - wesentlich durch diese heterosexuelle Ordnung geprägt ist (2014, S. 186). In der Beziehung zum Analytiker wird nach Laplanche die Beziehung zum Anderen im Sinne der Urverführung «reaktualisiert» (2011, S. 194), insofern als 
der Analytiker die Rolle der sexuellen Mutter als Urverführerin, Hüterin des Rätsels und Provokateurin der Übertragung einnimmt (Koellreuter, 2001, S. 145). An der so entstehenden «Geschichte» sind beide, Analytiker_in und Analysand_in als von gesellschaftlichen Ordnungssystemen tangierte sexuelle Subjekte mit einem grösstenteils unbewussten Begehren beteiligt. Die Schwierigkeit, die Michael Ermann für heterosexuelle Analytiker in der Arbeit mit homosexuellen Patienten beschreibt, dass sie «unbewusst an das Leitbild einer heterosexuellen Urszene gebunden» sind, «das sie nun an ihre Analysanden herantragen» (2009, S. 360), trifft daher nicht nur für diese spezifische Konstellation, sondern für jede psychoanalytische Situation zu. Die (unbewusste) Angst, sich als Analytiker_in mit der eigenen Geschlechts- und sexuellen Konstitution sowie damit einhergehender (Un-)Gewissheiten auseinandersetzen zu müssen, dürfte einer der wichtigsten Gründe dafür sein, dass viele Kolleg_innen Trans*-, aber nach wie vor auch homo- und bisexuelle Patient_innen, lieber an «auf diese Fragen spezialisierte» Kolleg_innen weiterverweisen.

\section{Reinszenierungen im Gruppenkontext}

Die eingangs beschriebene Szene spielte sich im gruppen- und milieutherapeutischen Setting einer psychotherapeutischen Tagesklinik ab. Dieses kann als gesellschaftlicher Mikrokosmos betrachtet werden, in dem Menschen unterschiedlichen Alters, sozialer Herkunft und Geschlechts zusammenkommen und sich multiple Übertragungs- und Gegenübertragungskonstellationen und -prozesse entfalten. Die gleichzeitige und stets miteinander verknüpfte therapeutische Bearbeitung gruppenbezogener wie individueller Dynamiken stellt für das darin involvierte Behandlungsteam eine Herausforderung dar, die eine sorgfältige Auseinandersetzung mit diesen Phänomenen erfordert. In einer Gruppe wird die Wirkmächtigkeit gesellschaftlicher Normalitätsvorstellungen auf eine nochmals andere Weise deutlich wie im Zweiersetting. Häufig werden bei Gruppenmitgliedern, die «aus dem heteronormativen Rahmen fallen» bzw. der Norm nicht zu entsprechen glauben, im Gruppenkontext Schamgefühle im Sinne von Konfrontations-, Ausschluss- und Sanktionsängsten noch verstärkt, was dazu führen kann, dass z. B. eine Teilnehmerin in der Gruppe nicht erzählt, dass sie eine Partnerin hat oder ein männliches Gruppenmitglied seine Liebeswünsche gegenüber einem Mann verschweigt. Ob solche Schwierigkeiten im Gruppenprozess zur Sprache kommen und bearbeitet werden können, hängt unserer Erfahrung nach wesentlich von der Sensibilisierung der Gruppenleiterin hinsichtlich der oben geschilderten Dynamikgesellschaftlicher - und gruppenbezogener - Normierungsund Diskriminierungsprozesse ab, die zwar subtil aber immer sehr wirksam vor sich 
gehen. Ebenso wichtig scheint uns allerdings, inwieweit ein Gruppenleiter auch im Hinblick auf die sich in der Gruppe abspielenden Interaktionen die hetero- und cis-normative Brille ablegen und sich selber auf das Spiel mehrgeschlechtlicher Identifizierungen und bisexueller Begehrensdynamiken einlassen kann. So werden beispielsweise Konflikte zwischen gleichgeschlechtlichen Teilnehmer_innen häufig unter dem Gesichtspunkt einer Konkurrenzthematik interpretiert. Was dabei aussen vor bleibt, ist das sexuell-triebhafte Geschehen in gleichgeschlechtlichen Beziehungen, das möglicherweise gerade dann zum Ausdruck kommt, wenn zwei sich in den Haaren liegen, in der ganzen Mehrdeutigkeit dieses Bildes.

Aus einer gruppenanalytischen Perspektive (vgl. Balmer, 2001) liesse sich fragen, inwiefern die eingangs geschilderte Ringkampffantasie der Gruppenleiterin primär eine Gegenübertragung auf Herrn S. als einzelnen Patienten war oder ob es sich vielmehr um eine Gegenübertragung auf die Gruppe als Ganzes handelte. Taucht eine Gegenübertragungsfantasie wie die geschilderte auf, so ist sie stets auch eine Einladung, über das Gruppengeschehen nachzudenken, selbst dann, wenn sie sich manifest auf einen einzelnen Patienten bezieht. Die Frage, was abgewehrt wird, stellt sich nicht nur für den Einzelnen, sondern stets auch auf der Ebene der Gruppe. Vordergründig war die Gruppe beschäftigt mit dem Einfordern von Versorgung, gegenseitigem Ratschläge-Erteilen und der Unzufriedenheit über das Behandlungsteam. Dabei war die Rollenverteilung klar «gendered»: Die weiblichen Gruppenteilnehmerinnen erschienen als diejenigen, denen geholfen werden musste. Die männlichen, in der Rolle der aktiv Ratschläge-Erteilenden, fokussierten auf die «hilflos-armen» Frauen, wehrten ihre eigene Bedürftigkeit ab und vermieden untereinander jegliche Nähe. Die Therapeutin wurde in dieser polaren Genderordnung als passiv und inkompetent entwertet. Erst zu einem späteren Zeitpunkt war Raum für das, was zunächst abgewehrt wurde: die Verletzlichkeit der Männer, die Potenz der Frauen, die homophile Seite des Heterosexuellen sowie das, was sich in der Gegenübertragung der Gruppenleiterin manifestierte: eine lustvoll-triebhafte Neugier für- und aufeinander, die sich wenig darum schert, wer sich gemäss gesellschaftlicher Konventionen für wen triebhaft interessieren darf.

\section{Queere Gegenübertragung - Utopie?}

Morgenthaler hat unterschieden zwischen der «Sexualität» als sekundärprozesshaft und gesellschaftlich geformter Sexualorganisation und dem «nicht organisierten, frei flottierenden Sexuellen» im Sinne der polymorph-perversen primärprozesshaften Triebregungen (1987, S. 146). Rudolf Balmer beschreibt aus gruppenanalytischer Perspektive, wie diese Triebregungen sich «in jeder 
Gegenübertragung» zeigen. Wichtig sei, «dass das Erkennen und Verstehen dieser Regungen den analytischen Prozess vorantreibt. Agieren und Normieren (was auch eine Form des Agierens sein kann) behindern den Prozess» (2001, S. 47). Man könnte nun zusammenfassend sagen, dass es im Fall der beschriebenen Gruppensituation wesentlich war, dass die Therapeutin die in ihrer Gegenübertragung auftauchende sexuelle Fantasie trotz der damit einhergehenden Irritation nicht unter dem Druck der hetero- und cisnormativen Ordnung weggeschoben hat, sondern als Ausdruck abgewehrter Triebwünsche verstand. Sie liess der «schwulen Gegenübertragung» ihren Platz. Gerade das Beispiel zeigt jedoch, wie schwierig es ist, nicht normierend zu agieren: So sortiere ich (N.B) zwar das in der Begegnung mit dem Patienten Erlebte spontan nicht im üblichen Sinne heteronormativ (aus). Zugleich greife ich aber auf heteronormativ tradierte Kategorien zurück: Die Gegenübertragung interpretiere ich als «schwule Fantasie». Meinen Impuls, «es dem Patienten zu zeigen», als «phallisch». Später - nachträglich - imaginiere ich die beiden Ringenden als «Vater» und «Sohn». In der darauf folgenden gemeinsamen Auseinandersetzung interpretieren wir die auf den Vater gerichteten Wünsche des Patienten als «homoerotisch» und die Dynamik in der Gruppe als «homophile Seite des Heterosexuellen». Mit Morgenthaler und Dannecker lässt sich sagen: In unserer Vorstellung wird das polymorph-perverse Sexuelle, das sich in der Begegnung mit dem Patienten und der Gruppe zeigt, zu einer Sexualität, welche geprägt ist durch diejenige der psychoanalytischen und sozialwissenschaftlichen Disziplinen und Denktraditionen, in denen wir sozialisiert sind. In diesem Sinne wäre vielleicht die Bezeichnung der Gegenübertragung in dieser Situation als «queer» anstatt «schwul» eine Möglichkeit gewesen, das in ihr sich zeigende polymorph-perverse Moment präziser zu fassen.

Bereits bei Freud zeigt sich eine Schwierigkeit, mit der die Psychoanalyse unweigerlich konfrontiert ist: So hat bereits er trotz seiner teils ziemlich revolutionären Bemühungen, die menschliche Sexualität zu denken und bestehende gesellschaftliche Normierungen auch zu hinterfragen, dieselbe in seinem Werk schliesslich doch immer wieder reproduziert und verfestigt - mit schwerwiegenden Folgen, wie die Geschichte der Psychoanalyse lehrt. Sich zu «lösen von all den Bedeutungsrastern», das «Nicht-Verstehen» des Merkwürdigen, Befremdenden auszuhalten, wie Kläui es thematisiert, erscheint uns als wesentlich für das klinische Arbeiten - und zwar nicht nur mit homo- oder bisexuellen bzw. Transpersonen. Jenem Raum zu geben, was befremdet und irritiert, sicher geglaubte Kategorien (auf Patient_innen-wie Therapeut_innenseite) immer wieder auch zu durchque(e)ren, ist jedoch stets eine Herausforderung. Dies insofern, als die analytische Begegnung ebenso wenig losgelöst von der sie mitkonstituierenden gesellschaftlich dominie- 
renden hetero- und cis-normativen Matrix wie von der diese auch durchkreuzenden triebhaften Verstrickung der am Geschehen beteiligten polymorph-sexuellen Subjekte stattfinden kann.

Sexuelle Differenzen sind latent wie manifest zugleich relevant wie irrelevant, das zeigt unser Fallbeispiel deutlich. Gerade auf dem Feld der ÜbertragungsGegenübertragungsdynamik können sich die damit einhergehenden Brüche, Inkongruenzen und Konflikte entfalten. Als que(e)re ist die Gegenübertragung eher Realität als Utopie. Man könnte auch sagen: Sie bleibt für die Psychoanalyse ein unverzichtbarer (Ver-)Störfaktor.

\section{Literatur}

Balmer, R. (2001). Das Begehren des Gruppenanalytikers; Zur homosexuellen Gegenübertragung in der Gruppenanalyse. Jahrbuch für Gruppenanalyse, 7, 43-56.

Becker, S. (2005). Weibliche und männliche Sexualität. In I. Quindeau \& V.Sigusch (Hrsg.), Freud und das Sexuelle (S. 63-79). Frankfurt a. M.: Campus Verlag . Bettighofer, S. (2000) Übertragung und Gegenübertragung im therapeutischen Prozess. Stuttgart: Kohlhammer.

Böllinger, L. (2015). Zur gesellschaftlichen Konstruktion von Sexualität und neuen Beziehungsformen. Psyche 69 (7), 603-631.

Burgermeister N. \& Garcia Nuñez D. (2015). Queerfeministische Perspektiven in der Psychotherapie. Psychosozial 140 (2), 55-70.

Butler, J. (1991 [1990]). Das Unbehagen der Geschlechter. Frankfurt a. M.: Suhrkamp. Dannecker, M. (2005). Männliche und weibliche Sexualität. In I. Quindeau \& V. Sigusch (Hrsg.), Freud und das Sexuelle (S. 80-94). Frankfurt: Campus.

Ermann, M. (2009). Das homosexuelle Dilemma. Zur Entwicklungsdynamik der normalen männlichen Homosexualität. Forum der Psychoanalyse 25 (4), $249-261$.

Freud, S. (2000 [1905]). Drei Abhandlungen zur Sexualtheorie. Studienausgabe. Bd. $V$ (S. 37-146). Frankfurt a. M.: Fischer.

Freud, S. (2000 [1912]). Zur Dynamik der Übertragung. Studienausgabe. Ergänzungsband (S. 157-168). Frankfurt a. M.: Fischer.

Garcia Nuñez, D. \& Jäger, M. (2011). Die sexuelle Gretchenfrage: homo- und bisexuelle Menschen im ärztlichen Setting. Swiss Medical Forum 11 (12), 213-217.

Gschwind, H. (2015). Psychoanalyse und Homosexualität. Psyche 69 (7), 632-647. 
Gsell, M. \&Zürcher, M. (2011). Licht ins Dunkel der Bisexualität. Bisexualität, anatomische Geschlechtsdifferenz und die psychoanalytische Bedeutung von männlich und weiblich, Psyche 65, (8), 669-729.

Gysling, A. (1995). Die analytische Antwort. Eine Geschichte der Gegenübertragung in Form von Autorenportraits. Tübingen: edition diskord.

Heenen-Wolff, S. (2015). Normativität in der Psychoanalyse - Eine Kritik. Psyche 69 (7), 585-602.

Heimann, P. (1950). On Counter-Transference. International Journal of PsychoAnalysis, 31, 81-84.

Kläui, C. (2008). Psychoanalytisches Arbeiten. Für eine Theorie der Praxis. Bern: Hans Huber.

Koellreuter, A. (2000). Das Tabu des Begehrens. Zur Verflüchtigung des Sexuellen in Theorie und Praxis der feministischen Psychoanalyse. Giessen: Psychosozial-Verlag.

Körner, J. (1990). Übertragung und Gegenübertragung, eine Einheit im Widerspruch. Forum der Psychoanalyse 6, 87-104.

Langiardi V. \& Capozzi P. (2004). Psychoanalytic attitudes towards homosexuality: An empirical research. Int J Psychoanal, 85, 137-158.

Laplanche, J. \& Pontalis, J.-B. (1975 [1967]). Das Vokabular der Psychoanalyse. Frankfurt a. M.: Suhrkamp.

Laplanche, J. (2011 [1987]). Neue Grundlagen für die Psychoanalyse. Giessen: Psychosozial.

Mertens, W. (1991). Einführung in die psychoanalytische Therapie (Bd. 3). Stuttgart: Kohlhammer.

Morgenthaler, F. (1987). Homosexualität, Heterosexualität, Perversion. Frankfurt a. M.: Fischer.

Passett, P. (2014 [2004]). Die anthropologische Dimension der Sexualität. Das Konzept der Sexualität im Rahmen der allgemeinen Verführungstheorie Jean Laplanches. In L. Bayer \& I. Quindeau (Hrsg.), Die unbewusste Botschaft der Verführung. Interdisziplinäre Studien zur Verführungstheorie Jean Laplanches (S. 139-169). Giessen: Psychosozial.

Poluda-Korte, E. (1993a). Sexualität in der Gegenübertragung. Zeitschrift für Sexualforschung 6 (3), 189-198.

Poluda-Korte, E. (1993b). Der «lesbische Komplex». Das homosexuelle Tabu und die Weiblichkeit. In E. M. Alves (Hrsg.) (1993). Stumme Liebe. Der «lesbische Komplex» in der Psychoanalyse. Freiburg: Kore. 
Poluda, E. (2000). Das Bild der lesbischen Frau in der Psychoanalyse. Psyche 54 (4), 322-353.

Phillips, S. H. (2003). Homosexuality: Coming out of the confusion. Int J Psychoanal 84, 1431-1450.

Quindeau, I. (2008). Verführung und Begehren. Die psychoanalytische Sexualtheorie nach Freud. Stuttgart: Cotta'sche Buchhandlung Nachfolger GmbH.

Quindeau, I. (2014). Lust auf Anderes. Die Implantation der heterosexuellen Ordnung in der allgemeinen Verführungstheorie. In L. Bayer \& I. Quindeau (Hrsg.), Die unbewusste Botschaft der Verführung. Interdisziplinäre Studien zurVerführungstheorie Jean Laplanches (S. 170-193). Giessen: Psychosozial.

Quindeau, I. (2015). Der Umgang mit dem homophoben Erbe. Psyche 69 (7), 648-660.

Racker, H. (2002 [1948-1958]). Übertragung und Gegenübertragung: Studien zur psychoanalytischen Technik. München: Reinhardt.

Rauchfleisch, U., Frossard, J., Waser, G., Wiesendanger, K. \& Roth, W. (2002). Gleich und doch anders: Psychotherapie und Beratung von Lesben, Schwulen, Bisexuellen und ihren Angehörigen. Stuttgart: Cotta'sche Buchhandlung Nachfolger GmbH.

Sellschopp, A. (1999). Primäre Femininität und das Geschlecht des Analytikers in Übertragung und Gegenübertragung. Psyche 53 (9/10), 1042-1070.

Sigusch, V. (1995). TranssexuellerWunsch und zissexuelle Abwehr. Psyche 49 (9/10), 811-837.

Sigusch, V. (2011). Zissexuelle und Transsexuelle: Über ein Neogeschlecht. In V. Zugig (Hrsg.), Auf der Suche nach der sexuellen Freiheit (S. 124-144). Frankfurt a. M./New York: Campus.

Stolorow, R., Branchaft, B. \& Atwood, G. E. (1996). Psychoanalytische Behandlung. Ein intersubjektiver Ansatz. Frankfurt a. M.: Fischer.

Thomä, H. (1999). Zur Theorie und Praxis von Übertragung und Gegenübertragung im psychoanalytischen Pluralismus. Psyche 53, (9/10), 820-872.

Zeul, M. (1993). Klinische Anmerkungen zur weiblichen Homosexualität. Psyche 47, (2), 107-129.

Zeul, M. (1999). Zwei Sprachen einer Körperphantasie. Zur Dynamik der Gegenübertragung. Psyche 53, (9/10), 1015-1041. 


\section{Anmerkungen}

1 Cis wird in diesem Kontext als Ergänzung bzw. Gegenstück verwendet. Als CisMenschen werden beispielsweise Personen bezeichnet, deren Geschlechtsidentität mit ihrem bei Geburt zugewiesenen Geschlecht übereinstimmt. Menschen, welche eine Differenz zwischen diesen beiden Grössen wahrnehmen, werden dementsprechend als Transpersonen bezeichnet. Analog können andere Begriffspaare wie Transfrau/Cisfrau, cisnormativ/transnormativ, usw. gebildet werden.

2 Um dieVielfalt unterschiedlicher Geschlechter und Geschlechtsidentitäten darstellen zu können, werden in diesem Text Pluralformen mit einem «Gender-Gap» (z. B. Patient_ innen) gekennzeichnet. Der Gender-Gap ist als Alternative zum Binnen-I (z. B. PatientInnen) zu verstehen, welches das Lesen und Denken zu binären Vorstellungen zwingt. Wo diese Schreibweise die Lesbarkeit erschwert, verwenden wir abwechslungsweise männliche/weibliche Formen.

3 Wenn man obiges Beispiel zur Anschauung nimmt, so stellt sich beispielsweise die Frage, wie die Therapeutin mit letzter Sicherheit entscheiden soll, ob ihr Gefühl der «Männlichkeit» durch den (unbewusst) vom Patienten initiierten Machtkampf, von ihrem (unbewusst heteronormativen) Eindruck, dass sie es ihm in der spiegelnden männlichen Identität am besten zeigen kann, oder von einem anderen eigenen, ihr nicht bewussten, inneren Konflikt ausgelöst wurde.

4 Der von Sigusch hier verwendete Begriff „,zissexuell“ entspricht dem von den Autor_ innen an Butler angelehnten Konzept des Cisnormativen.

\section{Angaben zur Autorin und zum Autor}

Nicole Burgermeister, Dr. phil., 1979, ist Soziologin, Klinische Psychologin, Psychoanalytikerin und Psychotherapeutin, Mitglied des Psychoanalytischen Seminars Zürich (PSZ) und in Ausbildung zur Gruppenanalytikerin am Seminar für Gruppenanalyse Zürich (SGAZ). Sie ist tätig in der Psychotherapeutischen Tagesklinik der Klinik Schützen, Rheinfelden, sowie in einer Praxisgemeinschaftspraxis in Zürich, wo die Arbeit mit LGBT-Klient_innen einen ihrer Schwerpunkte bildet.

David Garcia Nuñez, Dr. med., 1975, ist Facharzt für Psychiatrie und Psychotherapie und Sexualtherapeut, Ausbildung am Freud-Institut Zürich. Er leitete 2011-2014 die Sprechstunde für Gender-Dysphorie sowie die Sexualmedizinische Sprechstunde am Universitätsspital Zürich und ist aktuell Leiter des Kompetenz-Zentrums für Geschlechter-Varianz am Universitätsspital Basel. 